\title{
USO DOS FRACTAIS NA ANÁLISE DA FRAGMENTAÇ̃̃O DE UMA FLORESTA ATRAVÉS DE IMAGENS DE SATÉLITE
}

\author{
Fábio Minoru Yamaji ${ }^{1}$ \\ Christel Lingnau ${ }^{2}$ \\ Carlos Roberto Sanquetta ${ }^{3}$
}

\begin{abstract}
RESUMO
Esta pesquisa utilizou a geometria fractal para analisar o padrão da paisagem de uma Floresta Ombrófila Mista alterada. A partir da imagem classificada do Landsat-5 TM, onde foram definidas 8 classes, determinou-se os índices da dimensão fractal "D" e do parâmetro de Pareto "a" para cada tipologia. Com a análise fractal foi possível associar o padrão de cobertura com os índices calculados. Os resultados mostraram que os fragmentos de araucária são os maiores (apresentando um parâmetro

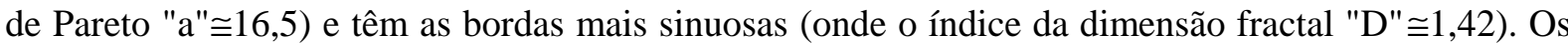
menores fragmentos são de capoeira ("a" $\cong 13,3)$ e os fragmentos com as bordas mais simples são da

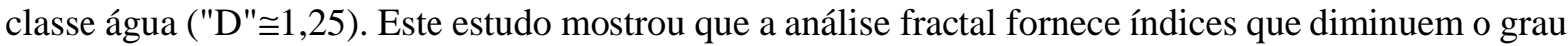
de subjetividade na avaliação da fragmentação da paisagem.
\end{abstract}

Palavras-chave: Padrões de paisagem, fragmentação, análise fractal, índice de paisagem

\section{USE OF FRACTALS IN FRAGMENTATION ANALYSIS OF MIXED ARAUCÁRIA FOREST BY SATELLITE IMAGES}

\begin{abstract}
This research aimed the use of fractals to analyze the landscape pattern of a Mixed Araucaria Forest. From the classified Landsat-5 TM image 8 classes were defined and then the fractal dimension "D" and the Pareto parameter "a", for each class, were determined. By using the fractal analysis it was possible to relate land cover pattern and estimated indices. The results showed that araucaria has the largest fragments (with Pareto parameter "a" $\cong 16,5$ ) and more complicated perimeters (where fractal

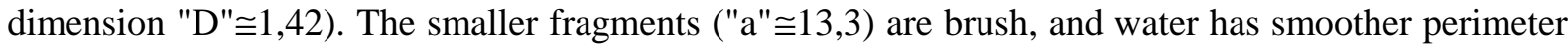

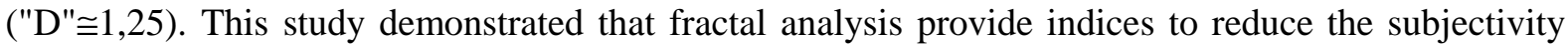
level in the evaluation of the landscape fragmentation.
\end{abstract}

Keywords: Landscape patterns, fragmentation, fractal analysis, landscape index

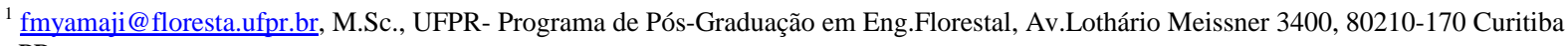
$-\mathrm{PR}$



3 sanqueta@ @loresta.ufpr.br, Dr., UFPR-Programa de Pós-Graduação em Engenharia Florestal Recebido paraa publicação: 07/2001

Aceito para publicação: 03/2002
} 


\section{INTRODUÇÃO}

De acordo com Turner e Gardner (1991), Lagro (1991) e Wiens (1995), o estudo da paisagem busca o entendimento de como a sua complexa estrutura espacial afeta os processos e os padrões da vegetação. Para isso, necessita de métodos para quantificar, identificar diferenças e determinar os padrões das paisagens. Entretanto, apesar de toda a atenção dispensada com relação a padrões espaciais, heterogeneidade e formas, poucos trabalhos têm sido executados sobre a análise da estrutura espacial desses mosaicos e seus efeitos ecológicos.

Uma das razões dessas características espaciais não serem muito empregadas são os próprios padrões determinados pela natureza que são inúmeros, e que não podem ser descritos por formas simples como, por exemplo, linhas, planos, ou cubos. A grande dificuldade encontrada nas análises espaciais era como definir ou quantificar todas essas formas irregulares descritas pela natureza. No entanto, desde que Mandelbrot introduziu a teoria dos fractais em 1975, a geometria fractal tem sido uma alternativa para a descrição desses padrões (Palmer,1988).

Assim sendo, este estudo utilizou os fractais para analisar uma paisagem florestal da região sul do Brasil com ocorrência da Floresta Ombrófila Mista alterada.

O objetivo foi, através da aplicação da análise fractal numa imagem classificada do satélite Landsat-5 TM, encontrar parâmetros que possam ser utilizados para o estudo da paisagem em uma área de Floresta Ombrófila Mista. O objetivo específico foi verificar a viabilidade da aplicação da dimensão fractal "D" e do parâmetro de Pareto "a", para as diferentes tipologias encontradas na área de estudo.

\section{REVISÃO BIBLIOGRÁFICA}

O conceito de dimensão fractal foi criado entre 1875 e 1925, mas foi só em 1975, com o polonês Benoit Mandelbrot, que se iniciou o estudo da geometria dos fractais. Ele criou a palavra fractal a partir do adjetivo latim fractus, que significa irregular ou quebrado (Barnsley et al., 1988).
Segundo Serra e Karas (1997), os fractais são figuras com propriedades e características peculiares, que os diferenciam das figuras geométricas habituais. Uma definição menos formal é dada por Palmer (1988), fractal é um objeto que tem detalhes em todas as escalas espaciais.

Conforme Barnsley et al. (1988), costuma-se pensar que os objetos naturais têm uma certa forma e que esta forma é determinada por uma escala característica como, por exemplo, um círculo, um quadrado ou uma figura geométrica qualquer. Para medir corretamente um objeto, como o seu comprimento, área ou volume, mede-se numa determinada escala e pode-se converter para qualquer outra escala. Essa idéia simples é baseada na geometria Euclidiana.

No entanto, Mandelbrot (1983) mostrou que muitos objetos não apresentam essa forma simplificada. Muitas estruturas não podem ser caracterizadas por uma única escala espacial, isto é, a forma depende da escala com que se analisa o objeto. Conforme ele mesmo cita, nuvens não são esferas e montanhas não são cones. Por exemplo, a copa de uma árvore numa determinada escala pode parecer um cone. No entanto, conforme se amplia esse objeto, novos detalhes são continuamente revelados e, quando se mede o seu comprimento, área ou volume, o valor encontrado depende do detalhamento da medição. Então, em adição à geometria Euclidiana existe uma outra geometria, fractal, que parece ser bem mais próxima da natureza.

Segundo Goodchild e Mark (1987);

DeJong e Burrough (1995), uma das características de todo o objeto fractal é a sua dimensão fractal, que é representada por "D". Esta é uma medida do grau de fragmentação. Os objetos naturais têm em comum uma forma extremamente irregular ou interrompida. Assim, é conveniente dizer que, certas curvas planas muito irregulares apresentam a sua dimensão fractal entre 1 e 2; em certas superfícies muito enrugadas e cheias de ondulações a sua dimensão fractal está entre 2 e 3.

Para determinar a dimensão fractal "D" não há uma metodologia padrão. Existem 
diferentes métodos para a obtenção da dimensão fractal "D". Entre eles pode-se citar os métodos variograma; contagem de boxes; prisma triangular; movimento Browniano; relação perímetro/área; e alguns desses modelos modificados. Segundo Verdyushkin (1994), matematicamente esses métodos são equivalentes.

O cálculo da dimensão fractal "D" permite fazer uma análise em termos de formas dos fragmentos gerados pela classificação. Essa análise é complementada pelo parâmetro de Pareto "a", que permite analisar uma outra característica dos fractais que é o fenômeno da escala, ou auto-similaridade; ou seja, a tendência de que os fractais não tenham uma característica ou tamanho típicos. Este fenômeno da escala permite descrever o tamanho da distribuição dos fragmentos (DeCola, 1989).

A geometria fractal tem se tornado uma importante ferramenta para análises de dados da paisagem. O'Neill et al. e Turner citados por Gaines et al. (1999) propuseram a dimensão fractal como um índice de medida para definição dos padrões da paisagem. Ainda, segundo os mesmos autores, a dimensão fractal descreve a

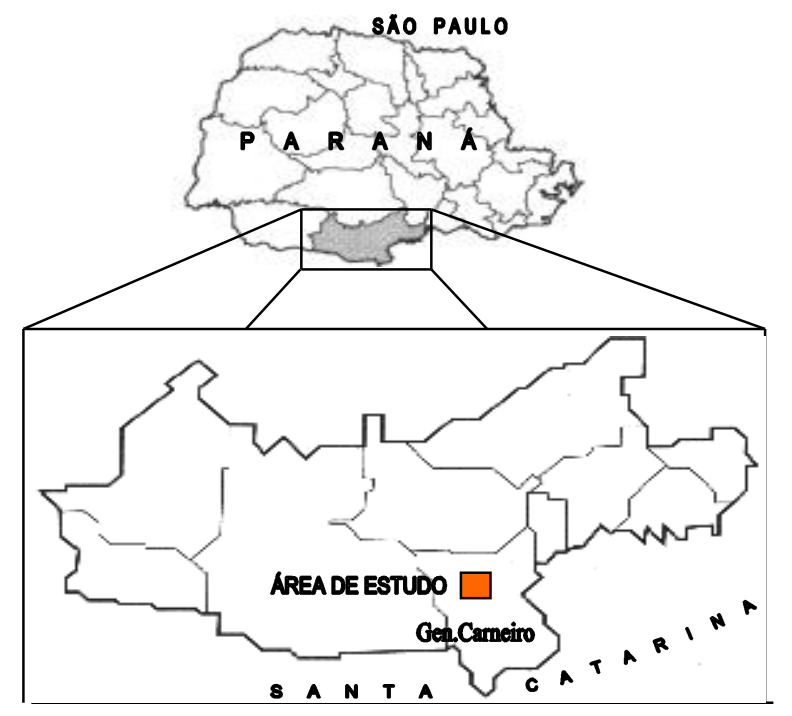

Figura 1: Mapa de localização da área de estudo.

forma do fragmento e a complexidade do contorno de uma paisagem.

Palmer (1988) explica que a vegetação é um exemplo típico de fractal, porque ela apresenta detalhes em todas as escalas espaciais de interesse. Numa escala grande, os padrões são definidos pelo espaçamento e interações individuais das plantas. Numa escala menor, a vegetação pode consistir de arranjos, que estão integrados com a paisagem. Numa escala menor ainda, os padrões de vegetação são influenciados por características geomorfológicas como vales, encostas, picos e corpos d'água.

\section{MATERIAL E MÉTODOS}

\section{Área de Estudo}

A área de estudo está localizada na Região Centro Sul do Paraná, no município de General Carneiro, a $280 \mathrm{~km}$ de Curitiba, de propriedade das Indústrias Pedro N. Pizzatto Ltda. (FIGURA 1). 
A área, que engloba as Fazendas São Pedro I, São Pedro II e Santa Cândida, estendese entre as coordenadas $26^{\circ} 20^{\prime} 35^{\prime}$ ' e $26^{\circ} 26^{\prime} 13^{\prime}$ ' Lat. S., e $51^{\circ} 19^{\prime} 49^{\prime \prime}$ e $51^{\circ} 25^{\prime} 29^{\prime}$ ' Long. W, num total de 4211 ha.

A formação vegetal encontrada nas áreas corresponde ao bioma denominado Floresta Ombrófila Mista.

A atual paisagem da área de estudo é o resultado das atividades florestais que se iniciaram na década de 30 . Os reflorestamentos foram realizados com uma técnica de dispersão dos talhões, mantendo-se faixas com espécies nativas formando os corredores ecológicos.

Para o trabalho foram utilizadas as imagens de satélite Landsat-5 TM, nas bandas 3, 4, 5 e 7, com resolução espacial de $30 \mathrm{~m}$; órbita/ponto 222-78, de 02/12/98.

Os cálculos para a análise fractal e o processamento da imagem foram executados fazendo-se uso do ambiente computacional e software mencionados na Tabela 1

Tabela 1: Software utilizado

\begin{tabular}{lll}
\hline Processamento da Imagem & Cálculos de Perímetro e Áreas & Cálculos Estatísticos e Gráficos \\
\hline ENVI 3.2 & \multirow{2}{*}{ ArcView 3.1 } & Excel Office 98 \\
ERDAS 8.4 & & Statistica 5 \\
\hline
\end{tabular}

Como dados complementares foi utilizado o Mapa do Brasil (IBGE) na escala de 1:100.000; folha SG-22-Y-B-III (União da Vitória), um mosaico de fotografias aéreas coloridas, obtidas em 1992 na escala de 1:10.000 e relatórios de Planos de Manejo e Inventário realizados para a empresa.

A definição das classes para a classificação supervisionada foi feita com base nos relatórios de manejo e inventário florestal existentes e complementadas com o trabalho de campo. Desta forma, foram definidas oito classes, as quais são:

- Água: inclui os rios, lagos, tanques, etc;

- Banhado: áreas com solo hidromórfico e com uma cobertura vegetal típica;

- Solo exposto: inclui os aceiros, pátios e as áreas ocupadas por construções;

- Capoeira: áreas com vegetação secundária, sem a presença de araucária ou folhosas de grande porte;

- Pinus: áreas com povoamentos homogêneos de Pinus sp.;

- Campo com araucária: áreas formadas por gramíneas e com araucárias dispersas;

- Folhosas com araucária: área de formação florestal mista onde o estrato superior é dominado pelas folhosas, com algumas araucárias esparsas;
- Araucária densa: área de formação florestal com domínio das araucárias no estrato superior com ocorrência de folhosas no estrato inferior.

A existência de dados sobre a área de estudo possibilitou a classificação supervisionada, que permite um maior controle sobre o processo de estratificação temática. Com relação ao algoritmo de classificação, foi utilizado o método "Máxima Verossimilhança".

Após a classificação, uma das etapas exigidas para a análise da dimensão fractal foi o cálculo da área e do perímetro de cada região que compõe as classes.

Entretanto, o pixel isolado é indesejável para o cálculo da dimensão fractal, uma vez que a relação perímetro-área resulta numa constante e, conseqüentemente, para qualquer classe o valor encontrado seria o mesmo. Para se eliminar os pixels isolados da imagem classificada foi aplicado o filtro de moda, utilizando-se de uma janela de $3 \times 3$ pixels.

A imagem classificada e filtrada foi então vetorizada e ficou composta por oito layers (cada layer correspondendo a uma classe). Esses layers foram exportados para o software ArcView, onde foram executados os cálculos das áreas e perímetros. As tabelas geradas foram complementadas no Excel com as respectivas transformações necessárias para se efetuar a 
regressão linear para determinar a dimensão fractal e o parâmetro de Pareto.

\section{Dimensão Fractal}

A partir da imagem classificada, filtrada e vetorizada, foi encontrada a dimensão fractal "D" para cada classe. Esse valor foi obtido pela regressão, através da relação entre o perímetro e a área de todos os fragmentos que formavam uma classe.

O cálculo da dimensão fractal é dado pela equação:

$p_{j}=c s_{j}^{(D / 2)}$

onde:

$p=$ perímetro do fragmento $j$

$c=$ coeficiente de forma

$s=$ área do fragmento $j$

$D=$ dimensão fractal da classe $i$

A equação (1) foi aplicada separadamente para cada classe, de forma que cada uma das oito classes teve um valor de dimensão fractal "D" específico. O valor da dimensão fractal "D" calculado deve refletir o padrão espacial de cada classe, onde $1 \bullet \mathrm{D} \bullet 2$. As classes cujos fragmentos apresentarem uma borda mais lisa deverão ter um valor de "D" próximo a 1, enquanto as classes cujos fragmentos tiverem uma borda mais sinuosa apresentarão um valor de $\mathrm{D}>1$, ou seja, próximo a 2.

Parâmetro de Pareto

Esta análise foi feita a partir da relação entre o fragmento e o seu grau (ou ranking). O grau de cada fragmento foi determinado pela comparação do seu tamanho com os demais de uma mesma classe (acrescidas de uma unidade). Assim sendo, foi estabelecido um ranking onde o maior fragmento de uma classe recebeu o grau dois, o segundo maior o grau três e assim sucessivamente até o último fragmento. Os cálculos foram feitos utilizando-se da relação hiperbólica dada pela seguinte equação:

$s_{j}=A r_{j}^{-b}$

onde:

$s=$ área do fragmento $j$

$A ; b=$ coeficientes $r=$ ranking ou grau do fragmento $j$

O parâmetro de Pareto "a" é encontrado pela linearização da equação (2), ou seja, ele é determinado pelo logaritmo neperiano do coeficiente A (parâmetro de Pareto "a" = ln A). A equação (2) foi aplicada separadamente para cada classe, de forma que cada classe teve um valor para o parâmetro de Pareto "a" específico.

\section{RESULTADOS E DISCUSSÃO}

\section{Dimensão Fractal "D"}

Através da regressão foi encontrado o valor da dimensão fractal "D" para cada uma das oito classes na imagem classificada. Os resultados com os valores da dimensão fractal "D" de cada classe e os respectivos coeficientes de correlação R2 podem ser vistos na TABELA 2.

Mesmo com algumas classes tendo apresentado um pequeno número de fragmentos (ver TABELA 2), o menor coeficiente de correlação encontrado para a regressão foi para a classe capoeira com $\mathrm{R} 2=0,97$. Estes resultados estão de acordo com Milne (1991), onde as estimativas da relação área-perímetro para fragmentos estão limitadas ao tamanho da janela utilizada na filtragem. Quanto maior o tamanho da janela utilizada maior será a perda dos detalhes na imagem. Portanto, os fragmentos pequenos estão mais sujeitos a erros de cálculo, porque são muito influenciados por um pequeno número de pixels.

\section{Parâmetro de Pareto "a"}

A relação existente entre a área e o ranking dos fragmentos resulta numa curva hiperbólica. Os resultados com os valores de "a" e os respectivos coeficientes de correlação R2 podem ser observados na TABELA 2. Para determinar o parâmetro de Pareto "a" utilizandose a equação (2), foram encontrados coeficientes de correlação acima de 0,90 para quase todas as classes, sendo os menores coeficientes de correlação $\mathrm{R} 2=0,84$ (campo com araucária) e $\mathrm{R} 2=0,90$ (água). 
Análise Fractal

Após os cálculos da dimensão fractal "D" e do parâmetro de Pareto "a", foram analisados, o número de pixels e o número de fragmentos de cada classe. Através desses dados, foi calculada a porcentagem que cada classe representa na imagem (área de estudo), tanto em pixels como em fragmentos. Todos esses dados foram colocados na TABELA 2, e formaram a base para a análise fractal.

Tabela 2: Dimensão fractal e parâmetro de Pareto para as oito classes de uso do solo

\begin{tabular}{lcccccc}
\hline \multicolumn{1}{c}{ classe } & \% Imagem & \% Fragmentos & $\mathrm{D}$ & $\mathrm{R}^{2}$ & $\mathrm{a}$ & $\mathrm{R}^{2}$ \\
\hline Água & 1 & 1,0 & 1,25 & 0,98 & 13,55 & 0,90 \\
Araucária densa & 42 & 23,5 & 1,40 & 0,98 & 16,50 & 0,97 \\
Banhado & 15 & 25,0 & 1,33 & 0,97 & 15,00 & 0,96 \\
Campo c/ araucária & 2 & 2,0 & 1,25 & 0,98 & 14,60 & 0,84 \\
Capoeira & 3 & 10,0 & 1,28 & 0,97 & 13,33 & 0,96 \\
Folhosa c/ araucária & 15 & 21,0 & 1,37 & 0,98 & 15,52 & 0,98 \\
Pinus & 15 & 9,0 & 1,33 & 0,98 & 16,57 & 0,96 \\
Solo exposto & 7 & 8,5 & 1,34 & 0,98 & 14,84 & 0,97 \\
& & & & & & \\
\hline
\end{tabular}

Uma análise da área de estudo pode ser feita observando-se as colunas \% Imagem e \% Fragmentos da TABELA2. A coluna \% Imagem mostra o quanto cada classe está contribuindo na formação da paisagem. Já a relação entre as duas colunas permite uma comparação entre os tamanhos médios dos fragmentos que compõem cada uma das oito classes definidas. A maior relação observada foi para a classe araucária densa que ocupa $42 \%$ da área de estudo com $23,5 \%$ dos fragmentos, indicando que essa classe é composta pelos maiores fragmentos. No outro extremo está a classe capoeira que apresenta a menor relação, ou seja, é formada pelos menores fragmentos. A classe folhosa com araucária constitui a segunda classe dominante, no entanto, em média, seus fragmentos têm, aproximadamente, $1 / 3$ do tamanho dos fragmentos da araucária densa. Somando-se as classes de folhosas com araucária, o pinus e a araucária densa, elas totalizam $72 \%$ da paisagem, mostrando que a paisagem é dominada por florestas.

A dimensão fractal "D" e o parâmetro de Pareto "a" plotados num gráfico de duas dimensões "forma x tamanho" (FIGURA 2), foram usados como uma técnica para avaliar a estrutura espacial das classes bem como a coerência dessa distribuição.

Através do gráfico, é possível fazer uma interpretação de como as classes estão distribuídas pela área. Observa-se que a classe de araucária densa domina a paisagem (maior valor de "D" e "a"). Isto é, as florestas de araucária densa são normalmente extensas e têm a borda bastante complexa (sinuosa). Ela contrasta com a classe água, geralmente menor e com a borda mais simples (lisa), onde $\mathrm{D}=1,25$. Esses resultados coincidem com aqueles obtidos por DeCOLA (1989), o qual encontrou para a floresta a maior dimensão fractal e a menor para a água.

A classe folhosa com araucária tem um valor de "D" relativamente alto $(\mathrm{D}=1,37)$, indicando que os fragmentos dessa classe apresentam bordas mais complexas (sinuosas) do que as classes capoeira $(D=1,28)$ e campo com araucária $(\mathrm{D}=1,25)$ que manifestam bordas mais lisas. 


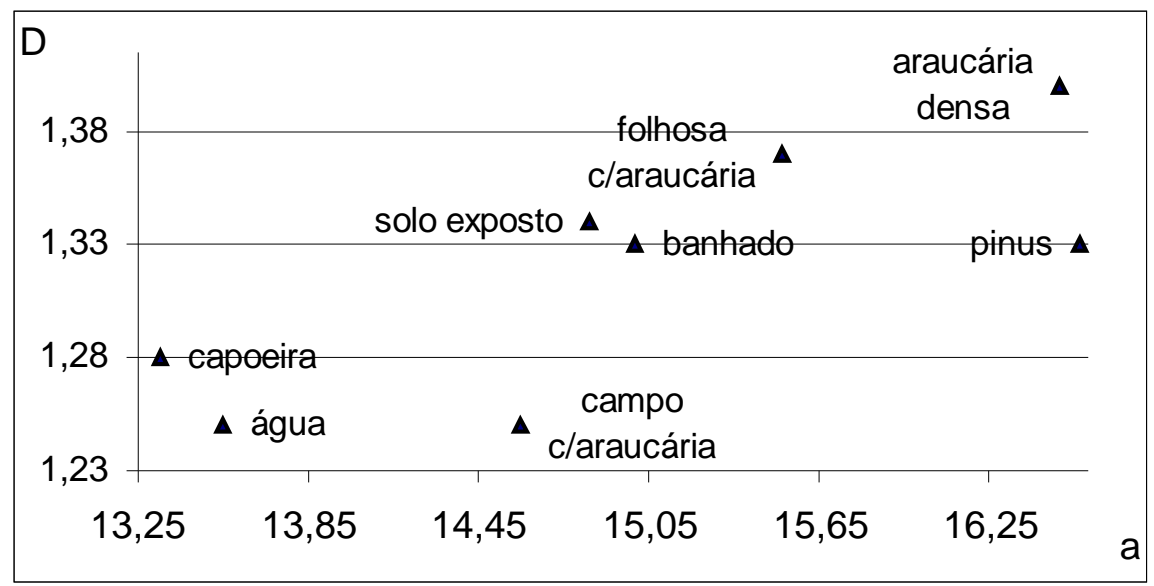

Figura 2: Gráfico da dimensão fractal "D" X parâmetro de Pareto "a" (valores médios)

Os valores da dimensão fractal "D" para a classe pinus encontrados nesse estudo $(D=1,33)$, podem ser considerados relativamente elevados quando comparado aos resultados obtidos por DeJong e Burrough (1995) e DeCola (1989). De acordo com esses autores, as regiões de povoamentos (pinus), tendem a ter um baixo valor de "D", refletindo as tendências das formas construídas pelo homem que normalmente criam as linhas e os blocos ou talhões (por exemplo, agricultura, povoamentos e áreas urbanas). Contudo, os elevados valores de "D" encontrados para a classe pinus, podem ser explicados pelo sistema de distribuição dos talhões adotado pela empresa. O sistema de formação de corredores ecológicos modificou a forma tradicional da distribuição dos talhões dos povoamentos em blocos. $\mathrm{Na}$ área de estudo, os talhões de pinus possuem contornos mais irregulares e estão mais fragmentados, portanto, resultando em valores de dimensão fractal "D" mais elevados que o esperado.

O maior valor do parâmetro de Pareto "a", foi observado para a classe pinus $(a=16,57)$ e para a classe araucária densa $(a=16,50)$, indicando que os fragmentos destas classes são os de maior tamanho na paisagem, enquanto que os fragmentos de capoeira $(a=13,33)$ são os menores. Isto pode ser explicado, porque os fragmentos de capoeira muitas vezes são decorrentes de áreas de pequenas lavouras abandonadas. Essas áreas trabalhadas pelo homem tendem a ter uma forma geométrica definida, que resulta num baixo valor de "D", e como são áreas pequenas, o parâmetro "a" também será baixo.

Esses resultados confirmam as observações, anteriormente feitas, através da análise da paisagem com base na TABELA 2.

\section{CONCLUSÃO}

Através da imagem do Landsat-5 TM, uma área de Floresta Ombrófila Mista alterada foi classificada em oito classes de uso do solo, as quais foram associadas aos padrões de cobertura através de medidas fractais.

Foi observado que os índices "D" e "a" podem ser usados separadamente, sendo que a dimensão fractal "D" exprime como estão as bordas dos fragmentos, enquanto o parâmetro de Pareto "a" trata do tamanho desses fragmentos. O cruzamento dos dois índices mostra como está a estrutura da paisagem, ou seja, as relações espaciais ("forma" x "tamanho") entre as classes.

Os resultados encontrados mostraram que os arranjos encontrados na área de estudo são compostos por florestas apresentando os maiores fragmentos e com as bordas mais sinuosas resultam nos maiores valores de "D", e no outro extremo estão as classes água, capoeira e campo como os menores fragmentos e com os índices de "D" mais baixos. Isto mostra que as três classes possuem fragmentos com a borda 
mais lisa, sendo que a água é resultado da margem (lisa) dos tanques e lagos; a capoeira é uma formação decorrente de pequenas lavouras (formas regulares) abandonadas e os campos são formações que, naturalmente têm a borda mais lisa.

A análise fractal aplicada no estudo da paisagem diminuiu o grau de subjetividade. As expressões como, "muito" ou "pouco" fragmentada, "grande" ou "pequeno" número de fragmentos, utilizadas para descrever uma paisagem, foram substituídas por índices da dimensão fractal "D" e do parâmetro de Pareto "a".

Este estudo explorou apenas um pequeno tópico da análise fractal, que foi a utilização do cálculo da dimensão fractal "D" e do parâmetro de Pareto "a". No entanto, os recursos e aplicações da geometria fractal, na área florestal, ainda não estão sendo muito utilizados. A análise fractal, com a sua capacidade descritiva poderá contribuir significativamente, para explicar a fragmentação e a dinâmica da paisagem florestal.

\section{RECOMENDAÇÕES}

Os índices fractais calculados foram para uma Floresta Ombrófila Mista, sendo a aplicação dos resultados limitada a uma região específica. Assim sendo, para uma análise mais completa desta formação, estudos em outras regiões são necessários, estabelecendo os parâmetros fractais para a flora e fauna.

É importante que a definição dos índices fractais também sejam estabelecidos para outras formações florestais, sendo recomendado:

a) determinar o valor dos índices da dimensão fractal "D" e do parâmetro de Pareto "a" considerados como "crítico", onde, a partir deste limite um fragmento ou determinada classe não encontraria condições para se desenvolver e tenderia a extinção;

b) associar os índices encontrados para a vegetação com índices determinados para a fauna, o que contribuirá para a integração do estudo fauna/flora;

c) determinar os índices da dimensão fractal "D" e do parâmetro de Pareto "a", em nível de espécies de interesse (fauna/flora); d) aplicar a análise fractal em imagens com outras resoluções (ex. IKONOS);

e) numa análise multi-temporal, que esses índices aplicados na interpretação da paisagem, indiquem como está evoluindo o processo de fragmentação.

\section{BIBLIOGRAFIA CITADA}

BARNSLEY, M.F.; DEVANEY, R.L.; MANDELBROT, B.B; PEITGEN, H.; SAUPE, D.; VOSS, R.F. (Ed.). The Science of Fractal Images. New York: Springer-Verlag, 1988.

DeCOLA, L. Fractal Analysis of a Classified Landsat Scene. Photogrametric Engineering and Remote Sensing, v.55, n.5, p.601-610, 1989.

DeJONG, S.M.; BURROUGH, P.A. A Fractal Approch to the Classification of Mediterranean Vegetation Types in Remotely Sensed Images. Photogrametric Engineering and Remote Sensing, v.61, n.8, p.1041-1053, 1995.

GAINES, W.L.; HARROD, R.J.; LEHMKUHL, J.F Monitoring Biodiversity: Quantification and Interpretation. Portland: PNW-GTR-433. U.S. Department of Agriculture, Forest Service, 1999. 27 p. General Technical Report.

GOODCHILD, M.F.; MARK, D.M. The Fractal Nature of Geographic Phenomena. Annals of the Association of American Geographers, v.77, n.2, p.265-278, 1987.

LaGRO Jr., J. Assessing Patch Shape in Landscape Mosaics. Photogrametric Engineering and Remote Sensing, v.57, n.3, p.285-293, 1991.

MANDELBROT, B.B. The fractal geometry of nature. New York: W.H. Freeman and Company, 1983.

MILNE, B.T. Lessons from applying fractal models to landscape patterns. In: TURNER, M.G.; GARDNER, R.H. (Ed.). Quantitative methods in landscpe ecology - The analysis and interpretation of landscape heterogeneity. New York: Spring-Verlag, 1991. p.199-235. 
PALMER, M.W. Fractal geometry: a tool for describing spatial patterns of plant communities. Vegetatio, v.75, p.91-102, 1988.

SERRA, C.P.; KARAS, E.W. Fractais, gerados por sistemas dinâmicos complexos. Curitiba: Champagnat, 1997.

TURNER, M.G.; GARDNER, R.H. (Ed.). Quantitative methods in landscpe ecology The analysis and interpretation of landscape heterogeneity. New York: Spring-Verlag, 1991.

VEDYUSHKIN, M.A. Fractal properties of forest spatial structure. Vegetatio, v.113, p.6570, 1994.

WIENS, J.A. Landscape mosaics and ecological theory. In: HANSSON, L.; FAHRIG, L.; MERRIAM, G. (Ed.). Mosaic landscape and ecological process. London: Chapman\&Hall, 1995. p.1-26. 